Nuno Ricardo Gonçalves Baptista Pereira ${ }^{1}$

Miguel Pedro da Rocha Branco 2

Maria Isabel Santos Silva ${ }^{3}$

Eulália Maria Bento Galhano 3

Raquel Maria Pereira Ortins Pina ${ }^{4}$

Luís Fllipe Costa Dias de Matos ${ }^{5}$

José Agostinho Valentim BARRos de Mesquita ${ }^{5}$

Relato de Caso

Palavras-chave

Hemangioma

Placenta/patologia

Pré-eclâmpsia

Gravidez múltipla

Keywords

Hemangioma

Placenta/pathology

Pré-eclampsia

Pregnancy, multiple

\section{Lesão vascular da placenta condicionando RCIU e hidropisia fetal não imune em gestação gemelar}

\section{Placental vascular lesion as cause of IUGR and nonimmune fetal} bydrops in twin pregnanc

\section{Resumo}

As lesões vasculares da placenta constituem um grupo de entidades distintas, mas inter-relacionadas, em que se incluem os corioangiomas e a corangiomatose multifocal difusa. $\bigcirc$ corioangioma é uma lesão nodular expansiva com incidência de cerca de $1 \%$. A corangiomatose multifocal difusa é rara $(0,2 \%)$ e predominante em placentas em idade gestacional inferior a 32 semanas. Os autores apresentam um caso de gestação gemelar monocoriônica/biamniótica, no qual um dos fetos, à $26^{a}$ semana de gestação, apresentou quadro de restrição de crescimento intrauterino, hidropisia e anemia associado à formação tumoral da placenta com vascularização aumentada verificada pela doplervelocimetria. $\bigcirc$ estudo anatomopatológico da placenta permitiu o diagnóstico de corangiomatose multifocal difusa. Este raro caso de corioangiomatose multifocal difusa com forma de apresentação pré-natal mimetizando a de um corioangioma comprova que a detecção ultrassonográfica de um tumor da placenta com vascularização aumentada deve suscitar outras hipótese diagnóstica, além do corioangioma.

\section{Abstract}

Placenta vascular lesions are a group of distinct yet related entities that include chorangiomas and diffuse multifocal chorangiomatosis. Chorangioma is an expansive nodular lesion with an incidence of about $1 \%$. Diffuse multifocal chorangiomatosis is rare $(0.2 \%)$ and mostly seen in placentas before the $32^{\text {nd }}$ gestational week. The authors present a case of a monochorionic/biamniotic twin pregnancy, in which, at the $26^{\text {th }}$ gestational week, one fetus developed intrauterine growth restriction (UGR), hydrops, and anemia associated with a tumor of the placenta with increased vascularization in the Doppler study. Pathological examination of the placenta diagnosed diffuse multifocal chorangiomatosis. This rare case report of diffuse multifocal chorangiomatosis with prenatal manifestations resembling those of a chorangioma proves that prenatal ultrasound detection of a placenta tumor, with increased vascularization at Doppler study, must raise other diagnostic possibilities beside chorangioma.
Correspondência:

Nuno Ricardo Goncalves Baptista Pereiro Avenida Doutor Armando Gonçalves 15 - apto. 207 3000-059 - Coimbra, Portugal

Recebido

$15 / 09 / 2011$
Trabalho realizado no Serviço de Obstetrícia da Maternidade Bissaya-Barreto - Coimbra, Portugal.

Interno Complementar de Ginecologia e Obstetrícia, Maternidade Bissaya-Barreto - Coimbra, Portugal

${ }^{2}$ Assistente de Ginecologia e Obstetrícia, Maternidade Bissaya-Barreto - Coimbra, Portugal.

${ }^{3}$ Assistentes Graduadas de Ginecologia e Obstetrícia, Maternidade Bissaya-Barreto - Coimbra, Portugal.

${ }^{4}$ Assistente Graduada de Anatomia Patológica, Serviço de Anatomia Patológica, Centro Hospitalar de Coimbra - Coimbra,

Portugal.

${ }^{5}$ Chefes do Serviço de Obstetrícia, Maternidade Bissaya-Barreto - Coimbra, Portugal. 


\section{Introdução}

Durante a gestação, a placenta constitui uma interface única entre a circulação sanguínea materna e a fetal, e o feto depende deste orgão no desempenho das funções hepática, pulmonar e renal ${ }^{1}$. Desta forma, a placenta assume um papel de especial importância nas gestações normais e de alto risco ${ }^{2}$.

Diversas condições, como a pré-eclâmpsia, síndrome antifosfolípido, doença hemolítica perinatal, doenças infecciosas e as lesões vasculares da placenta, podem interferir nos mecanismos de transporte útero-placentárioº As lesões vasculares da placenta constituem um grupo de entidades distintas, mas inter-relacionadas, tais como corioangiomas, corangiose, corangiomatose focal e corangiomatose multifocal difusa ${ }^{3,4}$.

O corioangioma é o tumor mais frequente da placenta, com uma incidência de aproximadamente $1 \%$ em séries com análise sistemática da placenta em secção $^{5-10}$. Contudo, a incidência daqueles de maiores dimensões (superior a $4 \mathrm{~cm}$ ) oscila entre 1:3500 e 1:9000 nascimentos ${ }^{9}$. Trata-se de uma lesão nodular vascular expansiva composta por capilares, estroma e trofoblasto, sendo mais frequente entre a $32^{\mathrm{a}}$ e a $37^{\mathrm{a}}$ semana de gestação e em placentas gemelares e de préeclâmpsia (PEC). Aqueles de pequenas dimensões têm pouca importância clínica. Entretanto, os de maior dimensão associam-se à restrição de crescimento intrauterino (RCIU), poli-hidrâmnios, trombocitopenia, coagulação intravascular disseminada (CID), diátese hemorrágica, hidropisia e morte fetal ${ }^{3,10,11}$. Discute-se a verdadeira natureza desta lesão, se neoplásica ou não, e ainda não há estudos clonais efetuados.

A corangiose é uma proliferação de capilares nas vilosidades terminais, caracterizadas histologicamente pela presença de pelo menos dez capilares em dez campos, observados com a objetiva de dez aumentos e em três áreas distintas da placenta ("regra dos dez") ${ }^{4}$. É uma lesão sem tradução macroscópica, apenas identificada histologicamente. É associada à hipóxia crônica pré-natal como em situações de PEC, anemia materna severa ou doença cardíaca grave, entre outras, e é mais frequente em placentas de termo, não se observando antes da $32^{\mathrm{a}}$ semana de gestação $0^{3,4}$.

A corioangiomatose é caracterizada pela proliferação de capilares nas vilosidades intermédias imaturas e nas principais, estando os vasos rodeados por fibras de reticulina que se misturam no estroma adjacente. Apresenta algumas características comuns com o coriangioma, nomeadamente a presença de células perivasculares, o aumento da celularidade e do colagéneo. Distinguem-se dois padrões principais, a localizada (focal ou segmentar) e a multifocal difusa. Esta envolve múltiplas áreas independentes da placenta. Geralmente não é uma lesão com tradução macroscópica, mas pode aparecer como nódulos dentro da placenta.

A corangiomatose multifocal difusa é relativamente rara $(0,2 \%)$ e predomina em placentas em idade gestacional inferior a 32 semanas $^{3,4,12}$. As placentas são grandes para a idade gestacional e demonstram vilosidades imaturas ${ }^{3}$. Fatores maternos, como idade avançada e antecedentes de mais do que cinco gestações anteriores, aumentam o risco de ocorrência deste tipo de lesão ${ }^{12}$. A corangiomatose multifocal difusa associa-se ainda à prematuridade, RCIU, PEC, gestação múltipla e anomalias congênitas ${ }^{3,4,10,12}$.

Os autores apresentaram um caso raro de corioangiomatose multifocal difusa com forma de apresentação pré-natal, mimetizando a de um corioangioma.

\section{Descrição do caso}

Paciente com 36 anos de idade, raça caucasiana, grávida 2 , para 0 , grupo sanguíneo A Rh positivo, com gestação gemelar monocoriônica/biamniótica espontânea. Dos antecedentes, destacava-se a presença de útero septado.

A gravidez foi acompanhada no hospital após a $13^{\mathrm{a}}$ semana, e decorreu sem intercorrências significativas até cerca da $26^{\mathrm{a}}$ semana. Dos exames realizados até o momento, salientam-se: ultrassonografia do primeiro trimestre, com translucência nucal normal em ambos os fetos; realização de amniocentese aproximadamente na $17^{\mathrm{a}}$ semana de gestação com o estudo citogenético revelando-se normal e estudo ultrassonográfico aproximadamente na $21^{\text {a }}$ semana de gestação, que demonstrou morfologia normal.

A ultrassonografia realizada na $26^{\text {a }}$ semana e a 4 dias da gestação (Tabela 1) revelou crescimento discrepante com o Feto 2 no percentil 5-10, com presença de derrame pericárdico e ligeira ascite, insuficiência da válvula tricúspide, velocidade do fluxo sanguíneo no pico sistólico na artéria cerebral média (PSV ACM) superior a 1,5 múltiplos da mediana e líquido amniótico reduzido (considerando normal após as 20 semanas de gestação, valores de coluna máxima vertical entre 2 e $10 \mathrm{~cm}$ ). Ambos os fetos apresentavam valores de índice de resistência da artéria umbilical (IRAU) normais ${ }^{13}$. A avaliação da estrutura placentária demonstrava uma formação nodular com $9 \mathrm{~cm}$ de maior diâmetro, com vascularização aumentada no estudo com Doppler de cor, sugerindo o diagnóstico de corioangioma (Figura 1).

Após os achados ultrassonográficos, realizou-se corticoterapia com betametasona para maturação pulmonar fetal. Foi solicitada a colaboração de um centro de referência com experiência na ablação vascular intraútero por via endoscópica, que, após avaliação do caso, aconselhou 
atitude conservadora com manutenção de rigorosa vigilância ultrassonográfica.

$\mathrm{Na} 27^{a}$ semana de gestação, a grávida foi internada em nossa unidade de Medicina Materno-fetal por elevação da pressão arterial. Durante a internação, manteve vigilância ultrassonográfica seriada, não tendo sido verificadas alterações significativas na fluxometria em ambos os fetos (sem evidenciarem sinais de centralização). A gestante desenvolveu um quadro de PEC severa, tendo sido realizada cesariana para extração fetal na $30^{a}$ semana e 5 dias de gestação. Durante o procedimento cirúrgico, confirmou-se a presença de septo uterino parcial.

Ambos os recém-nascidos (RN) eram do sexo masculino, o primeiro com $1.585 \mathrm{~g}$ (percentil 25 a 50) e índice de Apgar 9/10/10, e o segundo com $750 \mathrm{~g}$ (inferior ao percentil 5) e Apgar 5/7/8. O primeiro RN necessitou apenas de suporte ventilatório inicial, não apresentando intercorrências no período neonatal e com desenvolvimento psicomotor normal aos 24 meses de idade. O segundo $\mathrm{RN}$ apresentava anemia grave $(3,9 \mathrm{~g} / \mathrm{dL})$, como sugerido pela elevação pré-natal do PSV ACM, com necessidade de transfusão de concentrado de eritrócitos, necessitou de suporte ventilatório e desenvolveu um quadro de sépsis e hiperbilirrubinémia no período neonatal, que foi resolvido com a terapêutica médica instituída. Aos 24 meses de idade, apresentou ligeiro atraso do desenvolvimento psicomotor.

O estudo anatomopatológico da placenta revelou placenta gemelar monocoriônica/biamniótica com peso adequado à idade gestacional $(530 \mathrm{~g})$ e $22 \times 18 \times 3 \mathrm{~cm}$. Não se identificaram formações nodulares. Porém, a face materna da placenta e a superfície de corte apresentavam duas áreas distintas, uma medindo $15 \times 12 \mathrm{~cm}$ com aspecto congestivo (e que se encontrava em relação com a inserção do cordão do feto 1) e outra medindo $14 \times 9 \mathrm{~cm}$ com aspecto homogêneo, franjado e pálido (Figura 2). O cordão do feto 2 tinha inserção velamentosa e encontrava-se relacionado com a segunda área descrita. O estudo histológico da placenta mostrou, na área correspondente ao feto 1 , vilosidades coriais com desenvolvimento adequado e congestão. $\mathrm{Na}$ área correspondente ao feto 2, observou-se aspecto imaturo das vilosidades, com predomínio das vilosidades intermédias, que apresentavam proliferação vascular. A técnica de reticulina mostrou uma rede de fibras reticulínicas perivascular, que se misturava com o estroma do eixo da vilosidade. Com o estudo imunocitoquímico com o anticorpo anti-CD31 (marcador endotelial), evidenciou-se a proliferação vascular e a natureza multifocal da lesão (Figura 3, centro e direita). Com o anticorpo antiactina muscular lisa, evidenciou-se proliferação das células musculares lisas da parede dos vasos, formando-se uma rede que ocupa o eixo da vilosidade (Figura 3, à esquerda).

Estes aspectos permitiram o diagnóstico de corioangiomatose multifocal difusa.

Tabela 1. Ultrassonografia à $26^{a}$ semana e 4 dias de gestação

\begin{tabular}{lcc}
\hline & Fefo 1 & Fefo 2 \\
\hline PC (mm) & 257,6 & 219,9 \\
PA (mm) & 228,0 & 203,0 \\
CF (mm) & 52,0 & 43,0 \\
EPF (g) & $1110,0(p 75)$ & $698,0(p 5-10)$ \\
LA & Normal & Reduzido \\
IRAU & 0,7 & 0,8 \\
PSV ACM (cm/s) & 41,0 & 67,0 \\
\hline
\end{tabular}

PC: perímetro craniano; PA: perímetro abdominal; $\mathrm{CF}$ : comprimento do fêmur; EPF: estimativa de peso fetal; p: percentil; LA: líquido amniótico; IRAU: índice de resistência da artéria umbilical; PSV ACM: velocidade do fluxo sanguíneo no pico sistólico na artéria cerebral média.

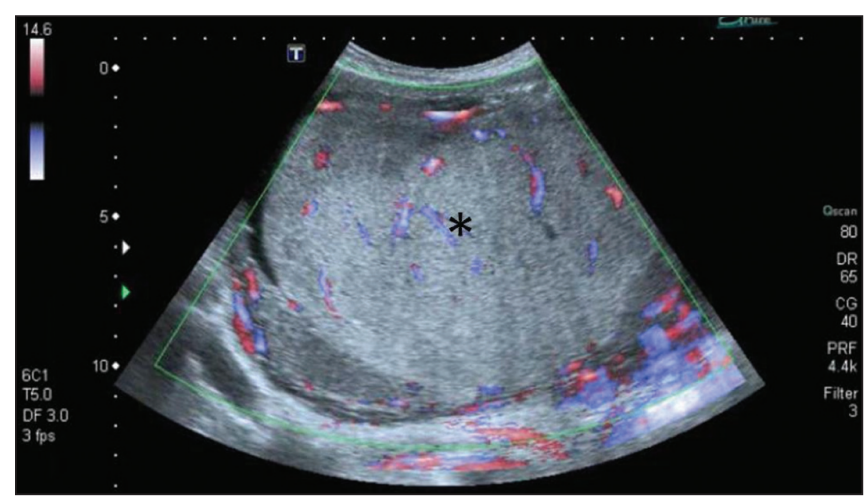

Figura 1. Formação nodular da placenta com $9 \mathrm{~cm}$ de maior diâmetro e com ecogenecidade que contrasta com o restante da placenta, com vascularização aumentada no estudo com Doppler de cor lo asterisco assinala a área correspondente à formação nodular).

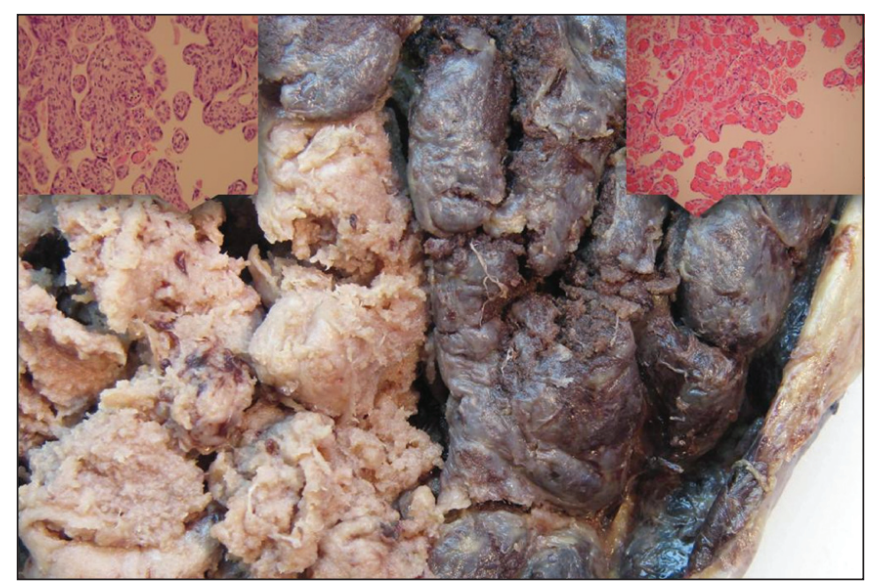

Figura 2. Face materna da placenta com área de aspecto franjado e pálida (esquerda - zona do feto 2) e área congestiva (direita - zona do feto 11. Nos cantos superiores observa-se o aspecto histológico das respectivas áreas coradas por H\&E, sendo possível verificar o aspecto imaturo das vilosidades, com predomínio das vilosidades intermédias e proliferação vascular na zona do feto 2, e vilosidades coriais com desenvolvimento adequado e congestão na zona do feto 1 


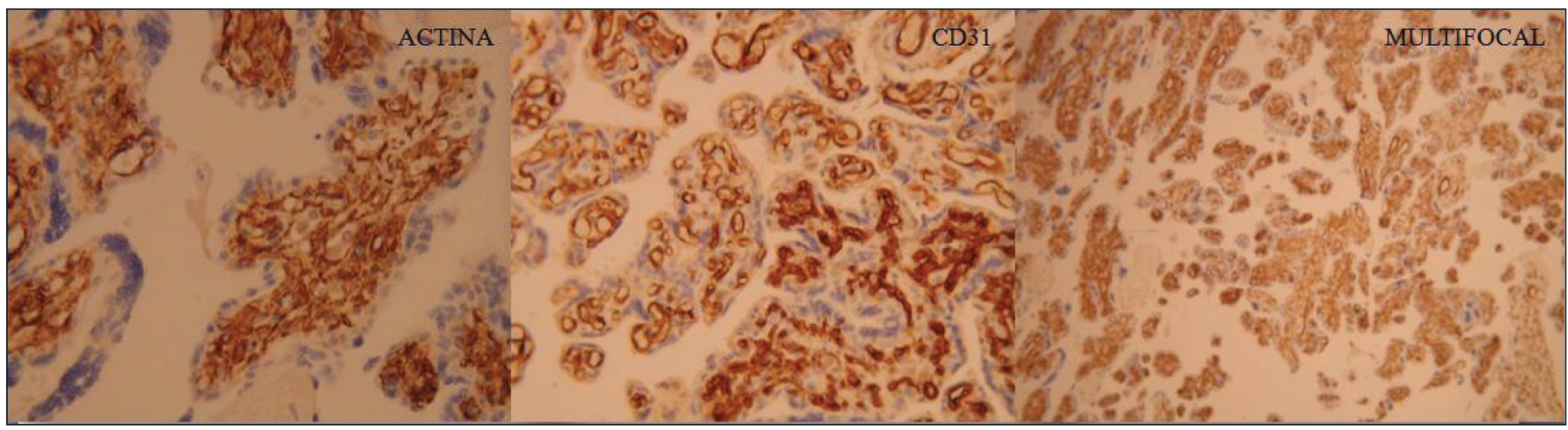

Figura 3. Estudo imunocitoquímico com marcador muscular liso (actina) evidenciou a proliferação das células musculares lisas da parede dos vasos, formando uma rede que ocupa o eixo da vilosidade (esquerda); o estudo com marcador endotelial (CD3 1 ) evidenciou a proliferação vascular e a natureza multifocal da lesão (centro e direita), na área da placenta correspondente ao feto 2.

\section{Discussão}

Atualmente, a ultrassonografia permite avaliar anomalias da placenta que podem influenciar a vigilância e o resultado das gestações ${ }^{8}$. O diagnóstico pré-natal de corioangioma enquadra-se neste contexto, uma vez que a ultrassonografia com doplervelocimetria permite o seu diagnóstico, bem como a sua distinção de outros tumores placentários, com benefícios no prognóstico materno-fetal ${ }^{2}$.

No caso ilustrado, a presença de um volumoso tumor da placenta, com vascularização aumentada, numa gestação gemelar monocoriônica/biamniótica condicionando RCIU, hidropisia fetal (derrame pericárdico e ascite) e anemia no feto, cujo cordão umbilical aparentava relação com essa área da placenta, suscitou o diagnóstico de corioangioma.

Acredita-se que a gênese deste tipo de lesão vascular tem por base a diminuição da tensão de oxigênio. A sua localização preferencial em locais de oxigenação reduzida, como a margem placentária ou sob a placa corial, bem como a sua incidência aumentada em populações que habitam em altitudes elevadas, são argumentos que favorecem esta hipótese ${ }^{3,4}$. A relevância clínica destes tumores relacionase diretamente com a sua dimensão, uma vez que aqueles maiores do que $4 \mathrm{~cm}$ associam-se ao efeito de shunt arteriovenoso com risco de perda fetal de aproximadamente 30 a $40 \%$, enquanto que os de pequena dimensão habitualmente não interferem com o decurso da gravidez ${ }^{2,3,9}$. Pelo impacto negativo que estes tumores podem ter na evolução da gravidez, o diagnóstico pré-natal de corioangioma deve conduzir a uma estrita vigilância ultrassonográfica, como foi ressaltado no presente caso.

Após o nascimento, o estudo anatomopatológico da placenta diagnosticou corioangiomatose multifocal difusa. Esta lesão, embora distinta do corioangioma, partilha diversas características com o mesmo. Estão ambas relacionadas a situações de hipóxia e apresentam diversos aspectos em comum, nomeadamente a sua associação à gravidez múltipla, PEC e RCIU. Entretanto, a idade gestacional em que predominam é substancialmente diferente, pois os corioangiomas têm maior frequência entre a $32^{\mathrm{a}}$ e a $37^{\mathrm{a}}$ semanas e a corioangiomatose multifocal difusa é mais frequente antes da $32^{\mathrm{a}}$ semana de gestação $0^{3,4,12}$.

Não se encontram descritos na literatura casos de corioangiomatose multifocal difusa com apresentação pré-natal mimetizando a do corioangioma, o que torna este relato extremamente invulgar. A presença aparente de uma lesão tumoral na avaliação ultrassonográfica pré-natal, não confirmada no estudo da placenta, revela a possibilidade deste tipo de lesão simular a presença de um corioangioma, fato que poderá estar relacionado com a extensa dimensão da área afetada (aproximadamente metade do disco placentar). Desta forma, este caso comprova que a detecção ultrassonográfica de um tumor da placenta, com vascularização aumentada no estudo Doppler, deve suscitar outras hipótese diagnósticas além do corioangioma.

Este relato realçou a importância da placenta como orgão fundamental no desenrolar da gestação, bem como a relevância da sua avaliação na ultrassonografia pré-natal, permitindo o diagnóstico precoce de anomalias e possibilitando a adaptação da vigilância pré-natal com melhoria dos resultados materno-fetais.

\section{Agradecimentos}

À equipe médica da Unidade de Cuidades Intensivos Neonatais da Maternidade Bissaya-Barreto pela colaboração e pelas informações prestadas sobre a evolução pós-natal dos RN. 


\section{Referências}

1. Cunningham FG, Leveno KJ, Bloom SL, Hauth JC, Rouse DJ, Spong CY. Williams obstetrics. 23rd ed. New York: McGraw-Hill Professional; 2009. Chapter 3: Implantation, embryogenesis, and placental development; p. 36-77.

2. Mauad Filho F, Costa AG, Spara P, Ferreira AC, Freitas Júnior RAO, Sala $M M$, et al. Tumor placentário diagnosticado durante a gravidez: relato de caso. Rev Bras Ginecol Obstet. 2002;24(7):485-9.

3. Rumboldt T, Eberts PT. Disorders of fetal vascular development chorangioma, localized chorangiomatosis, chorangiosis, and diffuse multifocal chorangiomatosis. Pathol Case Rev. 2008;13(6): 236-40.

4. Ogino S, Redline RW. Villous capillary lesions of the placenta: distinctions between chorangioma, chorangiomatosis, and chorangiosis. Hum Pathol. 2000;31(8):945-54.

5. Baruteau J, Joomye R, Muller JB, Vinceslas C, Baraton L, Joubert $M$, et al. Chorioangiomatosis: a rare etiology of nonimmune hydrops fetalis. Obstetric and pediatric implications for patient care. Arch Pediatr. 2009;16(10):1341-5.

6. Fox H. Vascular tumors of the placenta. Obstet Gynecol Surv. 1967;22(5):697-711.
7. Hirata GI, Masaki DI, O'Toole M, Medearis AL, Platt LD. Color flow mapping and Doppler velocimetry in the diagnosis and management of a placental chorioangioma associated with nonimmune fetal hydrops. Obstet Gynecol. 1993;81 (5 Pt 2):850-2.

8. Kuhlmann RS, Warsof S. Ultrasound of the placenta. Clin Obstet Gynecol. 1996;39(3):519-34.

9. Quintero RA, Reich H, Romero R, Johnson MP, Gonçalves L, Evans MI. In utero endoscopic devascularization of a large chorioangioma. Ultrasound Obstet Gynecol. 1996;8(1):48-52.

10. Amer HZ, Heller DS. Chorangioma and related vascular lesions of the placenta: a review. Fetal Pediatr Pathol. 2010;29(4):199-206.

11. Mochizuki T, Nishiguchi $T$, Ito I, Imai $M$, Isoda $H$, Masui $T$, et al. Case report Antenatal diagnosis of chorioangioma of the placenta: MR features. J Comput Assist Tomogr. 1996;20(3):413-6.

12. Bagby C, Redline RW. Multifocal chorangiomatosis. Pediatr Dev Pathol. $2011 ; 14(1): 38-44$.

13. Acharya G, Wilsgaard T, Berntsen GK, Maltau JM, Kiserud T. Reference ranges for serial measurements of umbilical artery Doppler indices in the second half of pregnancy. Am J Obstet Gynecol. 2005; 192(3):937-44. 